\title{
LAGRANGIAN MINIMAL ISOMETRIC IMMERSIONS OF A LORENTZIAN REAL SPACE FORM INTO A LORENTZIAN COMPLEX SPACE FORM
}

\author{
BANG-YEN CHEN AND LUC VRANCKEN
}

(Received April 12, 2000)

\begin{abstract}
It is well-known that the only minimal Lagrangian submanifolds of constant sectional curvature $c$ in a Riemannian complex space form of constant holomorphic sectional curvature $4 c$ are the totally geodesic ones. In this paper we investigate minimal Lagrangian Lorentzian submanifolds of constant sectional curvature $c$ in Lorentzian complex space form of constant holomorphic sectional curvature $4 c$. We prove that the situation in the Lorentzian case is quite different from the Riemannian case. Several existence and classification theorems in this respect are obtained. Some explicit expression of flat minimal Lagrangian submanifolds in flat complex Lorentzian space form are also presented.
\end{abstract}

1. Introduction. Let $\tilde{M}_{k}^{n}(4 c)$ be an indefinite complex space form of complex dimension $n$ and complex index $k$. The complex index is defined as the complex dimension of the largest complex negative definite subspace of the tangent space. If $k=1$, we say that $\tilde{M}(4 c)$ is Lorentzian. The curvature tensor $\tilde{R}$ of $\tilde{M}(4 c)$ is given by

$$
\tilde{R}(X, Y) Z=c\{\langle Y, Z\rangle X-\langle X, Z\rangle Y+\langle J Y, Z\rangle J X-\langle J X, Z\rangle J Y+2\langle X, J Y\rangle J Z\} .
$$

Let $\boldsymbol{C}^{n}$ denote the complex number $n$-space with complex coordinates $z_{1}, \ldots, z_{n}$. The $\boldsymbol{C}^{n}$ endowed with $g_{s, n}$, i.e., the real part of the Hermitian form

$$
b_{s, n}(z, w)=-\sum_{k=1}^{s} \bar{z}_{k} w_{k}+\sum_{j=s+1}^{n} \bar{z}_{j} w_{j}, \quad z, w \in \boldsymbol{C}^{n},
$$

defines a flat indefinite complex space form with complex index $s$. We simply denote the pair $\left(\boldsymbol{C}^{n}, g_{s, n}\right)$ by $\boldsymbol{C}_{s}^{n}$. In particular, $\boldsymbol{C}_{1}^{n}$ is the flat Lorentzian complex $n$-space.

For complex space forms with nonzero curvature $c>0$, consider the differentiable manifold:

$$
S_{2}^{2 n+1}(c)=\left\{z \in C_{1}^{n+1} ; b_{1, n+1}(z, z)=1 / c\right\},
$$

which is an indefinite real space form of constant sectional curvature $c$. The Hopf fibration

$$
\pi: S_{2}^{2 n+1}(c) \rightarrow C P_{1}^{n}: z \mapsto z \cdot C^{*}
$$

is a submersion and there exists a unique pseudo-Riemannian metric of complex index one on $\boldsymbol{C} P_{1}^{n}$ such that $\pi$ is a Riemannian submersion. The pseudo-Riemannian manifold $\boldsymbol{C} P_{1}^{n}(4 c)$ is a Lorentzian complex space form of positive holomorphic sectional curvature $4 c$.

2000 Mathematics Subject Classification. Primary 53C40, 53D12; Secondary 53B25, 53C50. 
Analogously, if $c<0$, consider

$$
H_{2}^{2 n+1}(c)=\left\{z \in \boldsymbol{C}_{2}^{n+1} ; b_{2, n+1}(z, z)=1 / c\right\},
$$

which is an indefinite real space form of constant sectional curvature $c<0$. The Hopf fibration

$$
\pi: H_{2}^{2 n+1}(c) \rightarrow C H_{1}^{n}: z \mapsto z \cdot C^{*}
$$

is a submersion and there exists a unique pseudo-Riemannian metric of complex index 1 on $\boldsymbol{C} H_{1}^{n}$ such that $\pi$ is a Riemannian submersion. The pseudo-Riemannian manifold $\boldsymbol{C H} H_{1}^{n}(4 c)$ is a Lorentzian complex space form of negative holomorphic sectional curvature $4 c$.

A complete simply-connected Lorentzian complex space form $\tilde{M}_{1}^{n}(4 c)$ is holomorphically isometric to $\boldsymbol{C}_{1}^{n}, \boldsymbol{C} P_{1}^{n}(4 c)$, or $C H_{1}^{n}(4 c)$, according to $c=0, c>0$ or $c<0$, respectively.

A submanifold $M$ of $\tilde{M}_{1}^{n}(4 c)$ is called Lagrangian if the almost complex structure $J$ of $\tilde{M}_{1}^{n}(4 c)$ interchanges the tangent and the normal spaces. Clearly, a Lagrangian submanifold of $\tilde{M}_{1}^{n}(4 c)$ is $n$-dimensional. A Lagrangian submanifold of $\tilde{M}_{1}^{n}(4 c)$ is Lorentzian if and only if $M$ has (real) index one.

One of the most fundamental problems in the study of Lagrangian submanifolds is the classification of Lagrangian isometric immersions of real space forms into complex space forms. For Riemannian complex space forms, this problem have been studied in [CO, E, C1, $\mathrm{C} 2, \mathrm{C} 3, \mathrm{CDVV} 2]$. In particular, Ejiri [E] proved that every minimal Lagrangian submanifold of constant sectional curvature $c$ in a Riemannian complex space form of holomorphic sectional curvature $4 c$ is totally geodesic and that the only minimal Lagrangian isometric immersions of real space forms into Riemannian complex space forms are either totally geodesic or flat Lagrangian submanifolds in a complex projective space (see, also [CO]).

In Lorentzian case, the corresponding problem was solved in [KV1] and [KV2] for minimal Lagrangian submanifolds of constant sectional curvature $c$ in a Lorentzian complex space form $\tilde{M}_{1}^{n}(4 \bar{c})$ with $c \neq \bar{c}$. However, the method used in [KV1] and [KV2] relies heavily on the assumption: $c \neq \bar{c}$; hence the method of [KV1] and [KV2] does not apply to the most fundamental case; namely, minimal Lagrangian submanifolds of constant sectional curvature $c$ in a Lorentzian complex space form $\tilde{M}_{1}^{n}(4 c)$ of holomorphic sectional curvature $4 c$.

The purpose of this paper is to investigate the most fundamental case; namely, to classify minimal Lagrangian submanifolds of constant sectional curvature $c$ in a Lorentzian complex space form $\tilde{M}_{1}^{n}(4 c)$ of holomorphic sectional curvature $4 c$. In Section 3 we determine completely the second fundamental form of minimal Lagrangian submanifolds of constant sectional curvature $c$ in a Lorentzian complex space form $\tilde{M}_{1}^{n}(4 c)$. In Sections 4 and 5, we classify flat minimal Lagrangian submanifolds in $\boldsymbol{C}_{1}^{n}$. In the last section, we provide some explicit expression of flat minimal Lagrangian submanifolds in $\boldsymbol{C}_{1}^{n}$.

2. Preliminaries. Let $M$ be a Lagrangian submanifold of a Lorentzian complex space form $\tilde{M}_{1}^{n}(4 c)$. Denote by $\nabla$ and $\tilde{\nabla}$ the Levi Civita connection on $M$ and $\tilde{M}_{1}^{n}(4 c)$, respectively. 
Then the formulas of Gauss and Weingarten are given respectively by

$$
\begin{aligned}
\tilde{\nabla}_{X} Y & =\nabla_{X} Y+h(X, Y), \\
\tilde{\nabla}_{X} J Y & =-A_{J Y} X+D_{X} J Y,
\end{aligned}
$$

where $h, A$ and $D$ are the second fundamental form, the shape operator and the normal connection. It is well-known that, for each $Y \in T_{x} M$, the shape operator $A_{J Y}$ is a symmetric endomorphism of the tangent space $T_{x} M$.

Since $J$ is the almost complex structure, we deduce as in the Riemannian case that

$$
\begin{gathered}
D_{X} J Y=J \nabla_{X} Y, \\
A_{J Y} X=-J h(X, Y)=A_{J X} Y .
\end{gathered}
$$

The equations of Gauss, Codazzi and Ricci are given respectively by

$$
\begin{gathered}
\langle R(X, Y) Z, W\rangle=\left\langle A_{h(Y, Z)} X, W\right\rangle-\left\langle A_{h(X, Z)} Y, W\right\rangle \\
+c(\langle X, W\rangle\langle Y, Z\rangle-\langle X, Z\rangle\langle Y, W\rangle), \\
(\nabla h)(X, Y, Z)=(\nabla h)(Y, X, Z), \\
\left\langle R^{D}(X, Y) J Z, J W\right\rangle=\left\langle\left[A_{J Z}, A_{J W}\right] X, Y\right\rangle \\
+c(\langle X, W\rangle\langle Y, Z\rangle-\langle X, Z\rangle\langle Y, W\rangle),
\end{gathered}
$$

where $X, Y, Z, W$ (respectively, $\eta$ and $\xi$ ) are vector fields tangent (respectively, normal) to $M, R^{D}(X, Y)=\left[D_{X}, D_{Y}\right]-D_{[X, Y]}$, and $\nabla h$ is defined by

$$
(\nabla h)(X, Y, Z)=D_{X} h(Y, Z)-h\left(\nabla_{X} Y, Z\right)-h\left(Y, \nabla_{X} Z\right) .
$$

We need the following Existence and Uniqueness Theorems for later use.

THEOREM A. Let $\left(M^{n}, g\right)$ be an $n$-dimensional simply connected Lorentzian manifold and TM denote the tangent bundle of $M^{n}$. If $\sigma$ be a TM-valued symmetric bilinear form on $M$ satisfying

(1) $\langle\sigma(X, Y), Z\rangle$ is totally symmetric,

(2) $(\nabla \sigma)(X, Y, Z)=\nabla_{X} \sigma(Y, Z)-\sigma\left(\nabla_{X} Y, Z\right)-\sigma\left(Y, \nabla_{X} Z\right)$ is totally symmetric,

(3) $R(X, Y) Z=c(\langle Y, Z\rangle X-\langle X, Z\rangle Y)+\sigma(\sigma(Y, Z), X)-\sigma(\sigma(X, Z), Y)$,

then there exists a Lagrangian isometric immersion $L$ from $(M, g)$ into a complete simplyconnected Lorentzian complex space form $\tilde{M}_{1}^{n}(4 c)$ whose second fundamental form $h$ is given by $h(X, Y)=J \sigma(X, Y)$.

THEOREM B. Let $L_{1}, L_{2}: M \rightarrow \tilde{M}_{1}^{n}(4 c)$ be two Lagrangian isometric immersions of a Lorentzian manifold $M$ with second fundamental forms $h^{1}$ and $h^{2}$, respectively. If

$$
\left\langle h^{1}(X, Y), J L_{1 \star} Z\right\rangle=\left\langle h^{2}(X, Y), J L_{2 \star} Z\right\rangle,
$$

for all vector fields $X, Y, Z$ tangent to $M$, then there exists an isometry $\phi$ of $\tilde{M}_{1}^{n}(4 c)$ such that $L_{1}=L_{2} \circ \phi$.

These two theorems can be proved in a way similar to the Riemannian case given in [CDVV1, C1]. 
3. Shape operator of minimal Lagrangian submanifolds. The main purpose of this section is to completely determine the shape operators of minimal Lagrangian submanifolds of constant sectional curvature $c$ in a Lorentzian complex space form $\tilde{M}_{1}^{n}(4 c)$. In order to do so, we need several lemmas. First we give the following.

LEMMA 1. Let $M$ be a Lagrangian submanifold of a Lorentzian complex space form $\tilde{M}_{1}^{n}(4 c)$. Then $M$ is of constant sectional curvature $c$ if and only if the shape operators of $M$ commute, i.e., $\left[A_{J Z}, A_{J W}\right]=0$ for all $Z, W$ tangent to $M$.

ProOF. (2.3) and (2.7) imply

$$
\langle R(X, Y) Z, W\rangle=\left\langle\left[A_{J Z}, A_{J W}\right] X, Y\right\rangle+c(\langle X, W\rangle\langle Y, Z\rangle-\langle X, Z\rangle\langle Y, W\rangle),
$$

which yields the lemma.

Next, we recall the following result from [On, pp. 261-262].

LEMMA 2. A symmetric endomorphism $S$ of an $n$-dimensional vector space $V$ with a Lorentzian inner product ( , ) can be put into one of the following four forms:

$$
\begin{aligned}
& \text { I. } S \sim\left(\begin{array}{cccc}
a_{1} & & & \mathbf{0} \\
& a_{2} & & \\
& & \ddots & \\
\mathbf{0} & & & a_{n}
\end{array}\right), \quad \text { II. } S \sim\left(\begin{array}{ccccc}
a_{0} & 0 & & & \mathbf{0} \\
1 & a_{0} & & & \\
& & a_{3} & & \\
& & & \ddots & \\
\mathbf{0} & & & & a_{n}
\end{array}\right) \text {, } \\
& \text { III. } S \sim\left(\begin{array}{cccccc}
a_{0} & 0 & 0 & & & \\
0 & a_{0} & 1 & & & \\
-1 & 0 & a_{0} & & & \\
& & & a_{4} & & \\
& & & & \ddots & \\
& & & & & a_{n}
\end{array}\right) \text {, } \\
& \text { IV. } S \sim\left(\begin{array}{ccccc}
a_{0} & b_{0} & & & \\
-b_{0} & a_{0} & & & \\
& & a_{3} & & \\
& & & \ddots & \\
& & & & a_{n}
\end{array}\right)
\end{aligned}
$$

where $b_{0}$ is assumed to be nonzero. In cases $\mathrm{I}$ and $\mathrm{IV}, S$ is represented with respect to an orthonormal basis $\left\{E_{1}, \ldots, E_{n}\right\}$ satisfying $\left(E_{1}, E_{1}\right)=-1,\left(E_{i}, E_{j}\right)=\delta_{i j},\left(E_{1}, E_{i}\right)=0$ for $2 \leq i, j \leq n$, while in cases II and III the basis $\left\{E_{1}, \ldots, E_{n}\right\}$ is pseudo-orthonormal satisfying $\left(E_{1}, E_{1}\right)=0=\left(E_{2}, E_{2}\right)=\left(E_{1}, E_{i}\right)=\left(E_{2}, E_{i}\right)$, for $3 \leq i \leq n,\left(E_{1}, E_{2}\right)=-1$, and $\left(E_{i}, E_{j}\right)=\delta_{i j}$, otherwise.

The following lemma is fundamental in this article. 
LEMMA 3. Let M be a minimal Lagrangian submanifold of constant sectional curvature $c$ in a Lorentzian complex space form $\tilde{M}_{1}^{n}(4 c)$. Then, for any vector $v$ tangent to $M$ and any natural number $k$, we have $\operatorname{trace}\left(A_{J v}^{k}\right)=0$.

Proof. Since $M$ is assumed to be minimal, one has trace $\left(A_{J v}\right)=0$ for any $v \in T M$. Thus, $\operatorname{trace}\left(A_{J A_{J v} v}\right)=0$. Therefore, by applying (2.4) and Lemma 1, we have

$$
\begin{aligned}
\sum_{i=1}^{n} \varepsilon_{i}\left\langle A_{J v}^{2} E_{i}, E_{i}\right\rangle & =\sum_{i=1}^{n} \varepsilon_{i}\left\langle A_{J v} A_{J E_{i}} v, E_{i}\right\rangle=\sum_{i=1}^{n} \varepsilon_{i}\left\langle A_{J E_{i}} A_{J v} v, E_{i}\right\rangle \\
& =\sum_{i=1}^{n} \varepsilon_{i}\left\langle A_{J A_{J v} v} E_{i}, E_{i}\right\rangle=0
\end{aligned}
$$

for any orthonormal basis $\left\{E_{1}, \ldots, E_{n}\right\}$, which implies trace $A_{J v}^{2}=0$. Similarly, we have

$$
\begin{aligned}
\sum_{i=1}^{n} \varepsilon_{i}\left\langle A_{J v}^{k} E_{i}, E_{i}\right\rangle & =\sum_{i=1}^{n} \varepsilon_{i}\left\langle A_{J v}^{k-1} A_{J E_{i}} v, E_{i}\right\rangle=\sum_{i=1}^{n} \varepsilon_{i}\left\langle A_{J E_{i}} A_{J v}^{k-1} v, E_{i}\right\rangle \\
& =\sum_{i=1}^{n} \varepsilon_{i}\left\langle A_{J A_{J v}^{k-1} v} E_{i}, E_{i}\right\rangle .
\end{aligned}
$$

Thus, by induction we obtain $\operatorname{trace}\left(A_{J v}^{k}\right)=0$ for any natural number $k$.

LEMMA 4. Let M be a minimal Lagrangian submanifold of constant sectional curvature $c$ in a Lorentzian complex space form $\tilde{M}_{1}^{n}(4 c)$. Then, for any point $x \in M$, exactly one of the following three cases occurs:

(A) $\left\langle A_{J w} w, w\right\rangle=0$ for all $w \in T_{x} M$;

(B) there is a vector $v \in T_{x} M$ such that $\left\langle A_{J v} v, v\right\rangle=1$, but $\left\langle A_{J w}^{2} w, w\right\rangle=0$ for all $w \in T_{x} M$

(C) there is a vector $v \in T_{x} M$ such that $\left\langle A_{J v}^{2} v, v\right\rangle= \pm 1$, but $\left\langle A_{J w}^{3} w, w\right\rangle=0$ for all $w \in T_{x} M$.

Proof. For any fixed $w \in T_{x} M, A_{J}$ is a symmetric endomorphism of $T_{x} M$. Thus, according to Lemma 2, $A_{J w}$ can be put into one of the four forms mentioned in Lemma 2.

(I) If $A_{J w}$ can be put into a diagonal matrix given in case I of Lemma 2, then Lemma 3 with $k=2$ yields $\sum_{i=1}^{n} a_{i}^{2}=0$, which implies $a_{1}=\cdots=a_{n}=0$. Thus $A_{J w}=0$.

(II) If $A_{J w}$ can be put into the form of the matrix in case II, then we have

$$
A_{J w} E_{1}=a_{0} E_{1}+E_{2}, \quad A_{J w} E_{2}=a_{0} E_{2}, \quad A_{J w} E_{i}=a_{i} E_{i}, \quad i=3, \ldots, n .
$$

Thus

$$
A_{J w}^{2} E_{1}=a_{0}^{2} E_{1}+2 a_{0} E_{2}, \quad A_{J w}^{2} E_{2}=a_{0}^{2} E_{2}, \quad A_{J w}^{2} E_{i}=a_{i}^{2} E_{i}, \quad i=3, \ldots, n .
$$


Hence, $0=\operatorname{trace}\left(A_{J w}^{2}\right)=2 a_{0}^{2}+a_{3}^{2}+\cdots+a_{n}^{2}$, which yields $a_{0}=a_{3}=\cdots=a_{n}=0$. Thus, in this case, $A_{J w}$ can be put in the form:

$$
A_{J w} \sim\left(\begin{array}{ccccc}
0 & 0 & & & \mathbf{0} \\
1 & 0 & & & \\
& & 0 & & \\
& & & \ddots & \\
\mathbf{0} & & & & 0
\end{array}\right) .
$$

In particular, we obtain $A_{J w}^{2}=0$ in case II.

(III) If $A_{J w}$ can be put into the matrix of case III, we have

$$
\begin{gathered}
A_{J w} E_{1}=a_{0} E_{1}-E_{3}, \quad A_{J w} E_{2}=a_{0} E_{2}, \quad A_{J w} E_{3}=E_{2}+a_{0} E_{3}, \\
A_{J w} E_{i}=a_{i} E_{i}, \quad i=4, \ldots, n, \\
A_{J w}^{2} E_{1}=a_{0}^{2} E_{1}-E_{2}-2 a_{0} E_{3}, \quad A_{J w}^{2} E_{2}=a_{0}^{2} E_{2}, \\
A_{J w}^{2} E_{3}=2 a_{0} E_{2}+a_{0}^{2} E_{3}, \quad A_{J w}^{2} E_{i}=a_{i}^{2} E_{i}, \quad i=4, \ldots, n .
\end{gathered}
$$

Hence, $0=\operatorname{trace}\left(A_{J w}^{2}\right)=3 a_{0}^{2}+a_{4}^{2}+\cdots+a_{n}^{2}$. Thus, in this case, $A_{J w}$ can be put in the form:

$$
A_{J w} \sim\left(\begin{array}{ccccc}
0 & 0 & 0 & & \mathbf{0} \\
0 & 0 & 1 & & \\
-1 & 0 & 0 & & \\
& & & \ddots & \\
\mathbf{0} & & & & 0
\end{array}\right) .
$$

In particular, we obtain $A_{J w}^{3}=0$.

(IV) If $A_{J w}$ can be put into the matrix of case IV, then we have

$$
\begin{gathered}
A_{J w} E_{1}=a_{0} E_{1}-b_{0} E_{2}, \quad A_{J w} E_{2}=b_{0} E_{1}+a_{0} E_{2}, \quad A_{J w} E_{i}=a_{i} E_{i}, \\
A_{J w}^{2} E_{1}=\left(a_{0}^{2}-b_{0}^{2}\right) E_{1}-2 a_{0} b_{0} E_{2}, \quad A_{J w}^{2} E_{2}=2 a_{0} b_{0} E_{1}+\left(a_{0}^{2}-b_{0}^{2}\right) E_{2}, \\
A_{J w}^{2} E_{i}=a_{i}^{2} E_{i}, \quad i=3, \ldots, n .
\end{gathered}
$$

Hence,

$$
\begin{gathered}
\operatorname{trace}\left(A_{J w}\right)=2 a_{0}+a_{3}+\cdots+a_{n}=z_{1}+z_{2}+a_{3}+\cdots+a_{n}=0, \\
\operatorname{trace}\left(A_{J w}^{2}\right)=2 a_{0}^{2}-2 b_{0}^{2}+a_{3}^{2}+\cdots+a_{n}^{2}=z_{1}^{2}+z_{2}^{2}+a_{3}^{2}+\cdots+a_{n}^{2}=0,
\end{gathered}
$$

where $z_{1}=a_{0}+i b_{0}, z_{2}=a_{0}-i b_{0}$. Similarly, we also have

$$
\operatorname{trace}\left(A_{J w}^{k}\right)=z_{1}^{k}+z_{2}^{k}+a_{3}^{k}+\cdots+a_{n}^{k}=0, \quad k=3,4, \ldots .
$$

From these we obtain $z_{1}=z_{2}=a_{3}=\cdots=a_{n}=0$. Thus, in this case we obtain $A_{J w}=0$. Consequently, we always have $A_{J w}^{3}=0$, which implies the lemma in a straightforward way. 
Equation (2.5) of Gauss implies that if $M^{n}(c)$ is a space-like minimal Lagrangian submanifold of constant sectional curvature $c$ in a Lorentzian complex space form $\tilde{M}_{1}^{n}(4 c)$ (or more generally, in an indefinite complex space form) of constant holomorphic sectional curvature $4 c$, then $M^{n}(c)$ is totally geodesic. Hence, from now on we only consider minimal Lagrangian Lorentzian submanifold of constant sectional curvature $c$ in $\tilde{M}_{1}^{n}(4 c)$.

The following main result of this section determines completely the second fundamental form for minimal Lagrangian submanifold of constant sectional curvature $c$ in a Lorentzian complex space form $\tilde{M}_{1}^{n}(4 c)$.

THEOREM 1. Let M be a minimal Lagrangian submanifold of constant sectional curvature $c$ in a Lorentzian complex space form $\tilde{M}_{1}^{n}(4 c)$. Then at every point $p$ the second fundamental form $h$ of $M$ takes one of the following three forms:

(1) $h=0$, i.e., $p$ is a totally geodesic point.

(2) The second fundamental form $h$ satisfies

$$
h\left(e_{1}, e_{1}\right)=J e_{2}, \quad h\left(e_{1}, e_{i}\right)=h\left(e_{i}, e_{j}\right)=0, \quad 2 \leq i, j \leq n
$$

with respect to a suitable pseudo-orthonormal basis $\left\{e_{1}, \ldots, e_{n}\right\}$ satisfying

$$
\begin{gathered}
\left(e_{1}, e_{1}\right)=0=\left(e_{2}, e_{2}\right)=\left(e_{1}, e_{i}\right)=\left(e_{2}, e_{i}\right), \quad\left(e_{1}, e_{2}\right)=1, \\
\left(e_{i}, e_{j}\right)=\delta_{i j}, \quad 1 \leq i, j \leq n-2 .
\end{gathered}
$$

(3) The second fundamental form $h$ satisfies

$$
h\left(e_{1}, e_{1}\right)=J e_{3}, \quad h\left(e_{1}, e_{3}\right)=J e_{2}, \quad \text { and } \quad h\left(e_{i}, e_{j}\right)=0, \quad \text { otherwise, }
$$

with respect to a suitable pseudo-orthonormal basis $\left\{e_{1}, \ldots, e_{n}\right\}$ satisfying (3.4).

Proof. The proof of this theorem bases on Lemma 4. Clearly, if case A of Lemma 4 occurs at every point $x$ in $M$, then $M$ is totally geodesic.

Let us assume that there is a point $x$ such that case B of Lemma 4 occurs, i.e., there is a vector $v \in T_{x} M$ such that $\left\langle A_{J v} v, v\right\rangle=1$ but $\left\langle A_{J w}^{2} w, w\right\rangle=0$ for all $w \in T_{x} M$. By linearity and Lemma 1 , the later condition implies $A_{J w}^{2}=0$ for all $w$.

In this case, we put $e_{1}=v$ and $e_{2}=A_{J v} v$. Then

$$
\left\langle e_{2}, e_{2}\right\rangle=\left\langle A_{J v} v, A_{J v} v\right\rangle=\left\langle A_{J v}^{2} v, v\right\rangle=0 .
$$

Moreover, from $\left\langle A_{J v} v, v\right\rangle=1$, we get $\left\langle e_{1}, e_{2}\right\rangle=1$.

For any real number $\alpha$ we have

$$
A_{J\left(e_{1}+\alpha e_{2}\right)}\left(e_{1}+\alpha e_{2}\right)=A_{J e_{1}} e_{1}+2 \alpha A_{J e_{1}} e_{2}+\alpha^{2} A_{J e_{2}} e_{2}=e_{2},
$$

where we use the facts: $A_{J e_{1}} e_{2}=A_{J v}^{2} v=0$ and $A_{J e_{2}} e_{2}=A_{J e_{2}} A_{J v} v=A_{J v} A_{J e_{2}} v=$ $A_{J v}^{2} e_{2}=0$. Thus, if we put $\alpha=-\left\langle e_{1}, e_{1}\right\rangle / 2$ and replace $e_{1}$ by $e_{1}+\alpha e_{2}$, we obtain for the new $e_{1}, e_{2}$ that

$$
\left\langle e_{1}, e_{1}\right\rangle=\left\langle e_{2}, e_{2}\right\rangle=0, \quad\left\langle e_{1}, e_{2}\right\rangle=1, \quad A_{J e_{1}} e_{1}=e_{2}, \quad A_{J e_{1}} e_{2}=0 .
$$

Clearly, both $\operatorname{Span}\left\{e_{1}, e_{2}\right\}$ and $\left\{e_{1}, e_{2}\right\}^{\perp}=:\left(\operatorname{Span}\left\{e_{1}, e_{2}\right\}\right)^{\perp}$ are invariant under the action of $A_{J e_{1}}$. Since $\left\{e_{1}, e_{2}\right\}^{\perp}$ is Riemannian, the restriction of $A_{J e_{1}}$ to $\left\{e_{1}, e_{2}\right\}^{\perp}$ is diagonalizable. 
Because $A_{J e_{1}}$ has zero as the only eigenvalue on $\left\{e_{1}, e_{2}\right\}^{\perp}$ (cf. proof of Lemma 4), the restriction of $A_{J e_{1}}$ to this invariant subspace is zero. Thus, if we choose $e_{3}, \ldots, e_{n}$ to be an orthonormal basis of $\left\{e_{1}, e_{2}\right\}^{\perp}$, we obtain

$$
A_{J e_{1}}=\left(\begin{array}{ccccc}
0 & 0 & & & \mathbf{0} \\
1 & 0 & & & \\
& & 0 & & \\
& & & \ddots & \\
\mathbf{0} & & & & 0
\end{array}\right)
$$

with respect to the pseudo-orthonormal basis $\left\{e_{1}, \ldots, e_{n}\right\}$.

From (2.4), (3.6) and Lemma 1, we find $A_{J e_{2}} e_{1}=A_{J e_{1}} e_{2}=0$ and $A_{J e_{2}} e_{2}=A_{J e_{1}}^{2} e_{2}=$ 0 , which imply that $\operatorname{Span}\left\{e_{1}, e_{2}\right\}$ is also invariant under the action of $A_{J e_{2}}$. Since $\left\{e_{1}, e_{2}\right\}^{\perp}$ is also invariant under $A_{J e_{2}}$, and $A_{J e_{2}}$ has zero as the only eigenvalue on $\left\{e_{1}, e_{2}\right\}^{\perp}$, we obtain $A_{J_{2}}=0$.

For $i=3, \ldots, n$, we have $A_{J e_{i}} e_{1}=A_{J e_{1}} e_{i}=0$, and $A_{J e_{i}} e_{2}=A_{J e_{i}} A_{J e_{1}} e_{1}=$ $A_{J e_{1}}^{2} e_{i}=0$. Thus, by applying the same argument as for $A_{J e_{2}}$, we also have $A_{J e_{i}}=0$ for $i=3, \ldots, n$. Consequently, in this case we obtain (3.3).

Next, we assume that there is a vector $v \in T_{x} M$ such that $\left\langle A_{J v}^{2} v, v\right\rangle= \pm 1$ but $\left\langle A_{J w}^{3} w, w\right\rangle$ $=0$ for all $w \in T_{x} M$. In this case, we put

$$
e_{1}=v, \quad e_{2}=A_{J e_{1}}^{2} e_{1}, \quad e_{3}=A_{J e_{1}} e_{1} .
$$

Then $\left\langle e_{1}, e_{2}\right\rangle= \pm 1$ and $A_{J e_{1}} e_{2}=0$, since $A_{J v}^{3} v=0$. Also, we have

$$
\begin{gathered}
\left\langle e_{3}, e_{3}\right\rangle=\left\langle A_{J e_{1}}^{2} e_{1}, e_{1}\right\rangle= \pm 1, \\
\left\langle e_{2}, e_{2}\right\rangle=\left\langle A_{J e_{1}}^{2} e_{1}, A_{J e_{1}}^{2} e_{1}\right\rangle=\left\langle A_{J e_{1}}^{4} e_{1}, e_{1}\right\rangle=0, \\
\left\langle e_{2}, e_{3}\right\rangle=\left\langle A_{J e_{1}} e_{3}, A_{J e_{1}}^{2} e_{1}\right\rangle=\left\langle A_{J e_{1}}^{3} e_{1}, e_{1}\right\rangle=0 .
\end{gathered}
$$

With respect to $e_{1}, e_{2}, e_{3}$, the restriction of the metric tensor $g$ to $\operatorname{Span}\left\{e_{1}, e_{2}, e_{3}\right\}$ takes the form:

$$
\left(\begin{array}{ccc}
* & \pm 1 & * \\
\pm 1 & 0 & 0 \\
* & 0 & \pm 1
\end{array}\right)
$$

If $\left\langle e_{3}, e_{3}\right\rangle=1$, then $\left\langle A_{J e_{1}}^{2} e_{1}, e_{1}\right\rangle=1$. Thus, $\left\langle e_{2}, e_{1}\right\rangle=1$. Similarly, if $\left\langle e_{3}, e_{3}\right\rangle=-1$, then $\left\langle e_{2}, e_{1}\right\rangle=-1$. Consequently, the restriction of the metric tensor $g$ to $\operatorname{Span}\left\{e_{1}, e_{2}, e_{3}\right\}$ takes one of the following two forms:

$$
B=\left(\begin{array}{ccc}
* & 1 & * \\
1 & 0 & 0 \\
* & 0 & 1
\end{array}\right) \quad \text { or } \quad C=\left(\begin{array}{ccc}
* & -1 & * \\
-1 & 0 & 0 \\
* & 0 & -1
\end{array}\right) .
$$

Since $\operatorname{det}(B)<0$, the signature of $B$ is either $(+,+,-)$ or $(-,-,-)$; while $\operatorname{det}(C)>0$, the signature of $C$ is either $(+,+,+)$ or $(-,-,+)$. Because $\operatorname{Span}\left\{e_{1}, e_{2}, e_{3}\right\}$ contains a null 
vector and $M$ is assumed to be Lorentzian, only the first case may occur. Thus, we must have $\left\langle e_{3}, e_{3}\right\rangle=\left\langle e_{1}, e_{2}\right\rangle=1$.

For any real numbers $\alpha, \beta$, we have

$$
\begin{gathered}
A_{J\left(e_{1}+\beta e_{2}+\alpha e_{3}\right)}\left(e_{1}+\beta e_{2}+\alpha e_{3}\right)=e_{3}+2 \alpha e_{2}, \\
A_{J\left(e_{1}+\beta e_{2}+\alpha e_{3}\right)}\left(2 \alpha e_{2}+e_{3}\right)=A_{J e_{1}} e_{3}, \\
\left\langle 2 \alpha e_{2}+e_{3}, 2 \alpha e_{2}+e_{3}\right\rangle=1 .
\end{gathered}
$$

Thus, if we replace $e_{1}, e_{3}$ by $e_{1}+\beta e_{2}+\alpha e_{3}, 2 \alpha e_{2}+e_{3}$, respectively, but keep $e_{2}$, then the new $e_{3}$ still satisfies $\left\langle e_{3}, e_{3}\right\rangle=1$.

Furthermore, since

$$
\begin{gathered}
\left\langle e_{1}+\beta e_{2}+\alpha e_{3}, e_{1}+\beta e_{2}+\alpha e_{3}\right\rangle=\left\langle e_{1}, e_{1}\right\rangle+\alpha^{2}+2 \alpha\left\langle e_{1}, e_{3}\right\rangle+2 \beta, \\
\left\langle e_{1}+\beta e_{2}+\alpha e_{3}, 2 \alpha e_{2}+e_{3}\right\rangle=\left\langle e_{1}, e_{3}\right\rangle+3 \alpha,
\end{gathered}
$$

so if we choose

$$
\alpha=-\frac{1}{3}\left\langle e_{1}, e_{3}\right\rangle, \quad \beta=\frac{5}{18}\left\langle e_{1}, e_{3}\right\rangle^{2}-\frac{1}{2}\left\langle e_{1}, e_{1}\right\rangle,
$$

then the new $e_{1}, e_{3}$ also satisfy $\left\langle e_{1}, e_{1}\right\rangle=\left\langle e_{1}, e_{3}\right\rangle=0$. By choosing an orthonormal basis $\left\{e_{4}, \ldots, e_{n}\right\}$ of $\left\{e_{1}, e_{2}, e_{3}\right\}^{\perp}$, we know that the metric tensor $g$ with respect to the pseudoorthonormal basis $\left\{e_{1}, \ldots, e_{n}\right\}$ is given by

$$
\left(\begin{array}{cccc}
0 & 1 & 0 & \\
1 & 0 & 0 & \mathbf{0} \\
0 & 0 & 1 & \\
& \mathbf{0} & & I_{n-3}
\end{array}\right),
$$

where $I$ denotes the identity matrix.

By applying $A_{J w}^{3}=0$ and the definition of $e_{1}, \ldots, e_{n}$ given above and proceeding as in the previous case, we obtain

$$
\begin{gathered}
A_{J e_{1}}=\left(\begin{array}{cccc}
0 & 0 & 0 & \\
0 & 0 & 1 & \mathbf{0} \\
1 & 0 & 0 & \\
& \mathbf{0} & & \mathbf{0}
\end{array}\right), \quad A_{J e_{3}}=\left(\begin{array}{cccc}
0 & 0 & 0 & \\
1 & 0 & 0 & \mathbf{0} \\
0 & 0 & 0 & \\
& \mathbf{0} & & \mathbf{0}
\end{array}\right), \\
A_{J e_{2}}=A_{J e_{4}}=\cdots=A_{J e_{n}}=0 .
\end{gathered}
$$

Thus, in this case the second fundamental satisfies (3.5).

4. Existence theorems. The purpose of this section is to show that there exist many minimal flat Lagrangian submanifolds in $\boldsymbol{C}_{1}^{n}$.

Let $\gamma=\gamma(t)$ be a null line in the Lorentzian $n$-space $L^{n}$ defined by $\gamma(t)=t \zeta$, where $\zeta$ is a (nonzero) null vector in $L^{n}$, and let $\alpha=\alpha(t)$ be a null curve lying in the hyperplane

$$
\Lambda^{n-1}=:\left\{x \in L^{n} ;\langle x, \zeta\rangle=1\right\}
$$

of $L^{n}$. Here by a null curve in $L^{n}$ we mean a curve $\alpha$ satisfying $\langle\alpha(t), \alpha(t)\rangle=0$ for any $t$. 
If $\alpha$ is a null line, we put $\varphi_{3}=\cdots=\varphi_{n}=0$ and choose $\left\{\alpha_{3}, \ldots, \alpha_{n}\right\}$ to be any fixed orthonormal basis of $\{\zeta, \alpha\}^{\perp}$.

If $\alpha$ is not a null line, then, by applying $\langle\alpha(t), \alpha(t)\rangle=0$ and $\langle\alpha(t), \zeta\rangle=1$, we know that $\alpha^{\prime}(t)$ is a vector perpendicular to $\alpha(t)$ and $\zeta$, i.e., $\left\langle\alpha^{\prime}(t), \alpha(t)\right\rangle=\left\langle\alpha^{\prime}(t), \zeta\right\rangle=0$. Thus, there is a unit vector $\alpha_{3}(t)$ and a real number $\varphi_{3}(t)$ such that $\alpha^{\prime}(t)=\varphi_{3}(t) \alpha_{3}(t)$. Since $\alpha$ is not a null line, it follows from $\langle\alpha(t), \zeta\rangle=1$ that $\zeta, \alpha$ and $\alpha^{\prime}$ are linearly independent.

If $n \geq 4$, then by using $\left\langle\alpha_{3}, \alpha_{3}\right\rangle=\langle\alpha, \zeta\rangle=1$ and $\left\langle\alpha, \alpha_{3}\right\rangle=0$, we know that $\alpha_{3}^{\prime}(t)=$ $-\varphi_{3}(t) \zeta+\varphi_{4}(t) \alpha_{4}(t)$, where $\varphi_{4}(t)$ is a real number and $\alpha_{4}(t)$ is a unit vector perpendicular to $\zeta, \alpha, \alpha_{3}$. When $\varphi_{4} \equiv 0$, we choose $\alpha_{4}, \ldots, \alpha_{n}$ to be any orthonormal basis of $\left\{\zeta, \alpha, \alpha_{3}\right\}^{\perp}$. Continuing this process if necessary, we obtain the Frenet curvatures $\varphi_{3}, \ldots, \varphi_{n}$ and the (orthonormal) Frenet frame $\left\{\alpha_{3}, \ldots, \alpha_{n}\right\}$ which satisfy the Frenet formulas:

$$
\left\{\begin{array}{l}
\alpha^{\prime}=\varphi_{3} \alpha_{3} \\
\alpha_{3}^{\prime}=-\varphi_{3} \zeta+\varphi_{4} \alpha_{4}, \\
\cdots \\
\alpha_{n-1}^{\prime}=-\varphi_{n-1} \alpha_{n-2}+\varphi_{n} \alpha_{n} \\
\alpha_{n}^{\prime}=-\varphi_{n} \alpha_{n-1} .
\end{array}\right.
$$

The main result of this section is the following existence theorems.

THEOREM 2. Suppose $\mu=\mu(t)$ is a function of one variable, $\gamma(t)=t \zeta$ a null line in the Lorentzian $n$-space $L^{n}$, and $\alpha=\alpha(t)$ a null curve lying in the hyperplane $\Lambda^{n-1}$ with Frenet curvatures $\varphi_{3}, \ldots, \varphi_{n}$ and the (orthonormal) Frenet frame $\left\{\alpha_{3}, \ldots, \alpha_{n}\right\}$ mentioned as above. Let $L^{n}$ be parameterized by

$$
x\left(t, u_{2}, \ldots, u_{n}\right)=t \zeta+u_{2} \alpha(t)+u_{3} \alpha_{3}(t)+\cdots+u_{n} \alpha_{n}(t) .
$$

Then, up to rigid motions, there exists a unique minimal Lagrangian isometric immersion $F_{\alpha \zeta \mu}: L^{n} \rightarrow C_{1}^{n}$ whose second fundamental form satisfies

$$
\left\{\begin{array}{l}
h\left(\frac{\partial}{\partial t}, \frac{\partial}{\partial t}\right)=\mu(t)\left(u_{3} \varphi_{3}(t)-1\right) J\left(\frac{\partial}{\partial u_{2}}\right), \\
h\left(\frac{\partial}{\partial t}, \frac{\partial}{\partial u_{i}}\right)=h\left(\frac{\partial}{\partial u_{i}}, \frac{\partial}{\partial u_{j}}\right)=0, \quad 2 \leq i, j \leq n .
\end{array}\right.
$$

Proof. It follows from (4.2) and a direct computation that the coefficients of metric tensor $g$ takes the form:

$$
\left(g_{i j}\right)=\left(\begin{array}{ccccc}
g_{11} & g_{12} & g_{13} & \ldots & g_{1 n} \\
g_{12} & 0 & 0 & \ldots & 0 \\
g_{13} & 0 & 1 & & 0 \\
\vdots & \vdots & & \ddots & \\
g_{1 n} & 0 & 0 & & 1
\end{array}\right)
$$


where

$$
\left\{\begin{array}{l}
g_{11}=u_{n-1}^{2} \varphi_{n}^{2}+\sum_{i=4}^{n}\left(u_{i-2} \varphi_{i-1}-u_{i} \varphi_{i}\right)^{2}, \\
g_{12}=1-u_{3} \varphi_{3}, \\
g_{13}=u_{2} \varphi_{3}-u_{4} \varphi_{4}, \\
\cdots \\
g_{1 n-1}=u_{n-2} \varphi_{n-1}-u_{n} \varphi_{n}, \\
g_{1 n}=u_{n-1} \varphi_{n} .
\end{array}\right.
$$

Thus, $\operatorname{det}\left(g_{i j}\right)=-g_{12}^{2}$ and

$$
\left(g^{i j}\right)=\frac{1}{g_{12}^{2}}\left(\begin{array}{ccccc}
0 & g_{12} & 0 & \ldots & 0 \\
g_{12} & -g_{11}+g_{13}^{2}+\cdots+g_{1 n}^{2} & -g_{12} g_{13} & \ldots & -g_{12} g_{1 n} \\
0 & -g_{12} g_{13} & g_{12}^{2} & & 0 \\
\vdots & \vdots & & \ddots & \\
0 & -g_{12} g_{1 n} & 0 & & g_{12}^{2}
\end{array}\right)
$$

By a straightforward computation, we obtain the Christoffel's symbols of $L^{n}$ as follows:

$$
\begin{aligned}
\Gamma_{11}^{1}= & -\frac{1}{g_{12}}\left(u_{3} \varphi_{3}^{\prime}+\varphi_{3} g_{13}\right), \quad \Gamma_{11}^{2}=-g^{22}\left(\varphi_{3} g_{13}+u_{3} \varphi_{3}^{\prime}\right), \\
\Gamma_{11}^{3}= & \frac{g_{13}}{g_{12}}\left(u_{3} \varphi_{3}^{\prime}+\varphi_{3} g_{13}\right)+u_{2} \varphi_{3}^{\prime}-u_{4} \varphi_{4}^{\prime}-\varphi_{4} g_{14}, \\
\Gamma_{11}^{k}= & \frac{g_{1 k}}{g_{12}}\left(u_{3} \varphi_{3}^{\prime}+\varphi_{3} g_{13}\right)+u_{k-1} \varphi_{k}^{\prime}-u_{k+1} \varphi_{k+1}^{\prime} \\
& +\varphi_{k} g_{1 k-1}-\varphi_{k+1} g_{1 k+1}, \quad 4 \leq k \leq n-1, \\
\Gamma_{11}^{n}= & \frac{g_{1 n}}{g_{12}}\left(u_{3} \varphi_{3}^{\prime}+\varphi_{3} g_{13}\right)+u_{n-1} \varphi_{n}^{\prime}+\varphi_{n} g_{1 n-1}, \\
\Gamma_{12}^{3}= & \varphi_{3}, \quad \Gamma_{12}^{1}=\Gamma_{12}^{2}=\Gamma_{12}^{4}=\cdots=\Gamma_{12}^{n}=0, \\
\Gamma_{13}^{1}= & -\frac{\varphi_{3}}{g_{12}}, \quad \Gamma_{13}^{2}=-g^{22} \varphi_{3}, \quad \Gamma_{13}^{3}=\frac{g_{13}}{g_{12}} \varphi_{3}, \quad \Gamma_{13}^{4}=\frac{g_{14}}{g_{12}} \varphi_{3}+\varphi_{4}, \\
\Gamma_{13}^{s}= & \frac{g_{1 s}}{g_{12}} \varphi_{3}, \quad 5 \leq s \leq n, \\
\Gamma_{14}^{3}= & -\varphi_{4}, \quad \Gamma_{14}^{1}=\Gamma_{14}^{2}=\Gamma_{14}^{4}=\Gamma_{14}^{6}=\cdots=\Gamma_{14}^{n}=0, \\
\Gamma_{1 r}^{1}= & \cdots=\Gamma_{1 r}^{r-2}=\Gamma_{1 r}^{r}=\Gamma_{1 r}^{r+2}=\cdots=\Gamma_{1 r}^{n}=0, \quad 5 \leq r \leq n \\
\Gamma_{1 r}^{r+1}= & \varphi_{r+1}, \quad \Gamma_{1 r+1}^{r}=-\varphi_{r+1}, \quad 4 \leq r \leq n-1, \\
\Gamma_{i j}^{k}= & 0, \quad 2 \leq i, j \leq n ; \quad 1 \leq k \leq n
\end{aligned}
$$


We define a symmetric bilinear form $\sigma$ on $M$ by

$$
\begin{aligned}
& \sigma\left(\frac{\partial}{\partial t}, \frac{\partial}{\partial t}\right)=\mu(t)\left(u_{3} \varphi_{3}(t)-1\right) \frac{\partial}{\partial u_{2}}, \\
& \sigma\left(\frac{\partial}{\partial t}, \frac{\partial}{\partial u_{i}}\right)=\sigma\left(\frac{\partial}{\partial u_{i}}, \frac{\partial}{\partial u_{j}}\right)=0, \quad 2 \leq i, j \leq n .
\end{aligned}
$$

From (4.2), (4.4), (4.5), (4.8) and $\langle\alpha, \alpha\rangle=0$, it follows that $\langle\sigma(X, Y), Z\rangle$ is totally symmetric in $X, Y$ and $Z$. Also, (4.4), (4.5), (4.7) and (4.8) show that $\sigma$ and the Riemannian curvature tensor $R$ of $L^{n}$ satisfy

$$
0=R(X, Y) Z=\sigma(\sigma(Y, Z), X)-\sigma(\sigma(X, Z) Y) .
$$

Furthermore, from (4.4), (4.5), (4.7) and (4.8) we obtain

$$
\begin{gathered}
\left(\nabla_{\frac{\partial}{\partial u_{s}}} \sigma\right)\left(\frac{\partial}{\partial t}, \frac{\partial}{\partial t}\right)=0=\left(\nabla_{\frac{\partial}{\partial t}} \sigma\right)\left(\frac{\partial}{\partial u_{s}}, \frac{\partial}{\partial t}\right), \quad s=2,4, \ldots, n, \\
\left(\nabla_{\frac{\partial}{\partial u_{3}}} \sigma\right)\left(\frac{\partial}{\partial t}, \frac{\partial}{\partial t}\right)=-\mu \varphi_{3} \frac{\partial}{\partial u_{2}}=\left(\nabla_{\frac{\partial}{\partial t}} \sigma\right)\left(\frac{\partial}{\partial u_{3}}, \frac{\partial}{\partial t}\right), \\
\left(\nabla_{\frac{\partial}{\partial u_{i}}} \sigma\right)\left(\frac{\partial}{\partial u_{j}}, \frac{\partial}{\partial t}\right)=\left(\nabla_{\frac{\partial}{\partial t}} \sigma\right)\left(\frac{\partial}{\partial u_{i}}, \frac{\partial}{\partial u_{j}}\right)=\left(\nabla_{\frac{\partial}{\partial u_{j}}} \sigma\right)\left(\frac{\partial}{\partial u_{i}}, \frac{\partial}{\partial t}\right)=0, \\
\left(\nabla_{\frac{\partial}{\partial u_{i}}} \sigma\right)\left(\frac{\partial}{\partial u_{j}}, \frac{\partial}{\partial u_{k}}\right)=0=\left(\nabla_{\frac{\partial}{\partial u_{j}}} \sigma\right)\left(\frac{\partial}{\partial u_{i}}, \frac{\partial}{\partial u_{k}}\right), \quad 2 \leq i, j, k \leq n .
\end{gathered}
$$

Hence, $(\nabla \sigma)(X, Y, Z)$ is also totally symmetric in $X, Y$ and $Z$. Consequently, by applying Theorems $\mathrm{A}$ and $\mathrm{B}$, we conclude that, up to rigid motions of $\boldsymbol{C}_{1}^{n}$, there exists a unique Lagrangian isometric immersion $F_{\alpha \zeta \mu}: L^{n} \rightarrow C_{1}^{n}$ whose second fundamental form satisfies (4.3).

THEOREM 3. Suppose $\mu(t), \lambda(t), \beta_{4}(t), \ldots, \beta_{n}(t)$ be $n-1$ functions of one variable, $\gamma(t)=t \zeta$ a null line in the Lorentzian $n$-space $L^{n}$, and $\alpha=\alpha(t)$ a null curve, not a null line, lying in the hyperplane $\Lambda^{n-1}$ with Frenet curvatures $\varphi_{3}, \ldots, \varphi_{n}$ and Frenet frame $\left\{\alpha_{3}, \ldots, \alpha_{n}\right\}$ as mentioned in Section 4 . Assume $L^{n}$ is parameterized by

$$
x\left(t, u_{2}, \ldots, u_{n}\right)=t \zeta+u_{2} \alpha(t)+u_{3} \alpha_{3}(t)+\cdots+u_{n} \alpha_{n}(t) .
$$

Then, up to rigid motions, there exists a unique minimal Lagrangian isometric immersion $I_{\alpha \zeta \mu \lambda \beta_{4} \cdots \beta_{n}}: L^{n} \rightarrow C_{1}^{n}$ whose second fundamental form satisfies

$$
\left\{\begin{array}{l}
h\left(\frac{\partial}{\partial t}, \frac{\partial}{\partial t}\right)=\beta_{2} J\left(\frac{\partial}{\partial u_{2}}\right)+\left(1-\varphi_{3} u_{3}\right) \sum_{i=3}^{n} \beta_{i} J\left(\frac{\partial}{\partial u_{i}}\right), \\
h\left(\frac{\partial}{\partial t}, \frac{\partial}{\partial u_{i}}\right)=\beta_{i} J\left(\frac{\partial}{\partial u_{2}}\right), \quad 3 \leq i \leq n \\
h\left(\frac{\partial}{\partial t}, \frac{\partial}{\partial u_{2}}\right)=h\left(\frac{\partial}{\partial u_{i}}, \frac{\partial}{\partial u_{j}}\right)=0, \quad 2 \leq i, j \leq n
\end{array}\right.
$$


where

$$
\begin{aligned}
\beta_{2}= & \sum_{j=4}^{n} \beta_{j}^{\prime} u_{j}+\sum_{k=5}^{n} \beta_{k}\left(\varphi_{k} u_{k-1}-\varphi_{k+1} u_{k+1}\right)-\beta_{4} \varphi_{5} u_{5} \\
& +\frac{\varphi_{3}^{\prime} u_{3}+\varphi_{3}^{2} u_{2}-\varphi_{3} \varphi_{4} u_{4}}{1-\varphi_{3} u_{3}} \sum_{j=4}^{n} \beta_{j} u_{j}+\left(1-\varphi_{3} u_{3}\right) \mu \\
& +\frac{\lambda}{1-\varphi_{3} u_{3}}\left\{\varphi_{3} u_{2}-\varphi_{4} u_{4}+\left(\frac{\varphi_{3}^{\prime}}{\varphi_{3}^{2}}\right)\left(2 \varphi_{3} u_{3}-1\right)\right\}+\frac{\lambda^{\prime}+\varphi_{4} \beta_{4}}{\varphi_{3}}
\end{aligned}
$$

and

$$
\beta_{3}=\frac{\lambda+\varphi_{3} \sum_{j=4}^{n} \beta_{j} u_{j}}{1-\varphi_{3} u_{3}} .
$$

PROOF. We define a symmetric bilinear form $\sigma$ on $M$ by

$$
\left\{\begin{array}{l}
\sigma\left(\frac{\partial}{\partial t}, \frac{\partial}{\partial t}\right)=\beta_{2}\left(\frac{\partial}{\partial u_{2}}\right)+\left(1-\varphi_{3} u_{3}\right) \sum_{i=3}^{n} \beta_{i}\left(\frac{\partial}{\partial u_{i}}\right), \\
\sigma\left(\frac{\partial}{\partial t}, \frac{\partial}{\partial u_{i}}\right)=\beta_{i}\left(\frac{\partial}{\partial u_{2}}\right), \quad 3 \leq i \leq n, \\
\sigma\left(\frac{\partial}{\partial t}, \frac{\partial}{\partial u_{2}}\right)=\sigma\left(\frac{\partial}{\partial u_{i}}, \frac{\partial}{\partial u_{j}}\right)=0, \quad 2 \leq i, j \leq n .
\end{array}\right.
$$

From (4.4), (4.5) and (4.14) it follows that $\langle\sigma(X, Y), Z\rangle$ is totally symmetric in $X, Y$ and $Z$. Also (4.4), (4.5), (4.7) and (4.14) show that $\sigma$ and the Riemannian curvature tensor $R$ of $L^{n}$ satisfy

$$
0=R(X, Y) Z=\sigma(\sigma(Y, X), X)-\sigma(\sigma(X, Z) Y) .
$$

Furthermore, from (4.5), (4.7), (4.14) and a straightforward long computation, we know that $(\nabla \sigma)(X, Y, Z)$ is also totally symmetric. Consequently, by applying Theorems $\mathrm{A}$ and $\mathrm{B}$, we conclude that, up to rigid motions of $\boldsymbol{C}_{1}^{n}$, there exists a unique Lagrangian isometric immersion $I_{\alpha \zeta \mu \lambda \beta_{4} \cdots \beta_{n}}: L^{n} \rightarrow C_{1}^{n}$ whose second fundamental form satisfies (4.11).

5. Classification of minimal flat Lagrangian submanifolds of $\boldsymbol{C}_{1}^{n}$. The purpose of this section is prove the following theorem which classify minimal flat Lagrangian submanifolds in $\boldsymbol{C}_{1}^{n}$.

THEOREM 4. Up to rigid motions of $\boldsymbol{C}_{1}^{n}$, locally (in a neighborhood of each point belonging to an open dense subset), every minimal Lagrangian isometric immersion $\Psi$ of $L^{n}$ into $C_{1}^{n}$ is either a $F_{\alpha \zeta \mu}$ given in Theorem 2 or a $I_{\mu \lambda \beta_{4} \cdots \beta_{n} \zeta \alpha}$ given in Theorem 3.

PROOF. Let $M$ be a minimal Lagrangian flat submanifold in $\boldsymbol{C}_{1}^{n}$ without totally geodesic points. Then, according to Theorem 1, the second fundamental form $h$ of $\Psi$ either takes 
the form:

$$
h\left(e_{1}, e_{1}\right)=J e_{2}, \quad h\left(e_{1}, e_{i}\right)=h\left(e_{i}, e_{j}\right)=0, \quad 2 \leq i, j \leq n
$$

with respect to a suitable pseudo-orthonormal frame $\left\{e_{1}, \ldots, e_{n}\right\}$ satisfying

$$
\begin{gathered}
\left(e_{1}, e_{1}\right)=0=\left(e_{2}, e_{2}\right)=\left(e_{1}, e_{i}\right)=\left(e_{2}, e_{i}\right), \quad\left(e_{1}, e_{2}\right)=1, \\
\left(e_{i}, e_{j}\right)=\delta_{i j}, \quad 1 \leq i, j \leq n-2,
\end{gathered}
$$

or takes the form:

$$
h\left(e_{1}, e_{1}\right)=J e_{3}, \quad h\left(e_{1}, e_{3}\right)=J e_{2}, \quad \text { and } \quad h\left(e_{i}, e_{j}\right)=0, \quad \text { otherwise },
$$

in a neighborhood of $p$, with respect to some pseudo-orthonormal basis $\left\{e_{1}, \ldots, e_{n}\right\}$ satisfying (5.2). It is clear that we can extend $\left\{e_{1}, \ldots, e_{n}\right\}$ to differentiable vector fields $\left\{E_{1}, \ldots, E_{n}\right\}$ on a neighborhood of $p$.

Let $\omega_{j}^{i}, 1 \leq i, j \leq n$ denote the connection forms with respect to $\left\{E_{1}, \ldots, E_{n}\right\}$, i.e.,

$$
\nabla_{X} E_{i}=\sum_{j=1}^{n} \omega_{i}^{j}(X) E_{j}
$$

We consider these two cases separately.

Case (I): Suppose that the second fundamental form takes the form (5.1).

In this case, (5.1), (5.2) and Codazzi equation (2.6) with $X=E_{i}, i \geq 2$, and $Y=Z=$ $E_{1}$ yield

$$
\begin{aligned}
& \omega_{2}^{1}\left(E_{i}\right)=\omega_{2}^{j}\left(E_{i}\right)=0, \quad 2 \leq i \leq n, \quad 3 \leq j \leq n, \\
& \omega_{2}^{2}\left(E_{i}\right)-2 \omega_{1}^{1}\left(E_{i}\right)=-\omega_{i}^{1}\left(E_{1}\right) .
\end{aligned}
$$

Also, from (5.1), (5.2) and Codazzi equation (2.6) with $Y=E_{i}, Z=E_{j}, i, j \geq 2$, and $X=E_{1}$, we know that $\nabla_{E_{i}} E_{j}$ has no components in $E_{1}$-direction. Thus, $E_{2}, \ldots, E_{n}$ span an integrable distribution, say $\mathcal{D}$, whose leaves are totally geodesic in $L^{n}$. Moreover, (5.4) implies that $\nabla_{E_{2}} E_{2} \in \operatorname{Span}\left\{E_{2}\right\}$. Hence, the integral curves of $E_{2}$ are pregeodesics of $L^{n}$.

Let

$$
\begin{aligned}
& F_{1}=E_{1}+b_{3} E_{3}+\cdots+b_{n} E_{n}-\frac{1}{2}\left(b_{3}^{2}+\cdots+b_{n}^{2}\right) E_{2}, \\
& F_{2}=E_{2}, \\
& F_{i}=E_{i}-b_{i} E_{2}, \quad i=3, \ldots, n,
\end{aligned}
$$

where $b_{3}, \ldots, b_{n}$ are functions on $L^{n}$. It is easy to see from (5.2) and (5.5) that $\left\{F_{1}, \ldots, F_{n}\right\}$ is also a pseudo-orthonormal frame satisfying (5.2). Moreover, (5.1) and (5.5) imply that the frame $\left\{F_{1}, \ldots, F_{n}\right\}$ preserves the same property for the second fundamental form $h$ of $\Psi$, that is, we have

$$
h\left(F_{1}, F_{1}\right)=J F_{2}, \quad h\left(F_{1}, F_{i}\right)=h\left(F_{i}, F_{j}\right)=0, \quad 2 \leq i, j \leq n .
$$

(5.6) and the discussion above show that the integral curves of $F_{2}$ are pregeodesics and the distribution spanned by $\left\{F_{2}, \ldots, F_{n}\right\}$ is an integrable distribution whose leaves are linear $(n-1)$-subspace of $L^{n}$. 
The above statement means that we can parameterize $L^{n}$ by

$$
x\left(t, v_{2}, \ldots, v_{n}\right)=\gamma(t)+\sum_{i=2}^{n} v_{i} \gamma_{i}(t),
$$

such that $\partial / \partial t=F_{1}$ and $\operatorname{Span}\left\{\partial / \partial v_{2}, \ldots, \partial / \partial v_{n}\right\}=\operatorname{Span}\left\{F_{2}, \ldots, F_{n}\right\}$.

Since $F_{1}$ is a null vector field, $F_{1}$ lies in the light cone. Thus, there exist suitable functions $b_{3}, \ldots, b_{n}$ such that $F_{1}$ is parallel to some constant null vector, say $\zeta$. In particular, this implies that an integral curve $\gamma=\gamma(t)$ of $F_{1}$ is a null line parallel to $\zeta$ in $L^{n}$. So, by reparameterizing if necessary, we may assume $\gamma(t)=t \zeta$ for some null vector $\zeta$.

Taking now $\tilde{F}_{2}$ as the multiple of $F_{2}$ such that $\left\langle\zeta, \tilde{F}_{2}\right\rangle=1$, it follows from (5.4) that

$$
\nabla_{F_{i}} \tilde{F}_{2}=0
$$

for $2 \leq i \leq n$. This means that in the $(n-1)$-dimensional subspace mentioned before, $\tilde{F}_{2}$ is a constant. It only changes along the integral curve of $F_{1}$. It follows that we may write

$$
x\left(t, u_{2}, \ldots, u_{n}\right)=t \zeta+u_{2} \alpha_{2}(t)+\cdots+u_{n} \alpha_{n}(t)
$$

where $\partial / \partial u_{2}=\tilde{F}_{2}$ and $\operatorname{Span}\left\{\partial / \partial u_{2}, \ldots, \partial / \partial u_{n}\right\}=\operatorname{Span}\left\{F_{2}, \ldots, F_{n}\right\}$ and thus $\alpha_{2}(t), \ldots$, $\alpha_{n}(t)$ satisfy

$$
\left\{\begin{array}{l}
\alpha_{2}(t)=b(t) E_{2}, \\
\left\langle\zeta, \alpha_{2}(t)\right\rangle=1 \\
\left\langle\alpha_{2}, \alpha_{2}\right\rangle=0 \\
\left\langle\zeta, \alpha_{i}(t)\right\rangle=\left\langle\alpha_{2}(t), \alpha_{i}(t)\right\rangle=0 \\
\left\langle\alpha_{i}(t), \alpha_{j}(t)\right\rangle=\delta_{i j}, \quad 3 \leq i, j \leq n
\end{array}\right.
$$

For simplicity, we may choose $\left\{\alpha_{3}, \ldots, \alpha_{n}\right\}$ to be the Frenet frame of the null curve $\alpha=\alpha_{2}(t)$ with the corresponding Frenet curvatures $\varphi_{3}, \ldots, \varphi_{n}$ as mentioned in Section 4.

From (5.7) and (5.8), we obtain

$$
\frac{\partial}{\partial t}=\zeta+u_{2} \alpha_{2}^{\prime}(t)+\cdots+u_{n} \alpha_{n}^{\prime}(t), \quad \frac{\partial}{\partial u_{2}}=b(t) E_{2},
$$

where $\zeta$ is a null vector parallel to $F_{1}$. Therefore, from (5.5), (5.6), (5.8) and (5.9) we get

$$
h\left(\frac{\partial}{\partial t}, \frac{\partial}{\partial t}\right)=\kappa J\left(\frac{\partial}{\partial u_{2}}\right), h\left(\frac{\partial}{\partial t}, \frac{\partial}{\partial u_{i}}\right)=h\left(\frac{\partial}{\partial u_{i}}, \frac{\partial}{\partial u_{j}}\right)=0,2 \leq i, j \leq n
$$

for some function $\kappa$.

From (5.10) and Codazzi equation (2.6) with $X=\partial / \partial u_{j}$ and $Y=Z=\partial / \partial t$, we obtain

$$
\frac{\partial \kappa}{\partial u_{j}}=\kappa\left(\Gamma_{j 1}^{1}-\Gamma_{j 2}^{2}\right), \quad j=2, \ldots, n .
$$


Using (4.7), equation (5.11) reduces to

$$
\begin{aligned}
& \frac{\partial \kappa}{\partial u_{2}}=\frac{\partial \kappa}{\partial u_{4}}=\cdots=\frac{\partial \kappa}{\partial u_{n}}=0, \\
& \frac{\partial \kappa}{\partial u_{3}}=\left(\frac{\varphi_{3}}{u_{3} \varphi_{3}-1}\right) \kappa .
\end{aligned}
$$

Solving (5.12) yields

$$
\kappa=\mu(t)\left(u_{3} \varphi_{3}-1\right)
$$

for some function $\mu(t)$. Hence, the second fundamental form of the immersion $\Psi$ satisfies (4.3). Thus, by applying Theorem B of uniqueness we conclude that, up to rigid motions of $\boldsymbol{C}_{1}^{n}$, the immersion is given by a $F_{\alpha \zeta \mu}$ associated with a null curve $\alpha$, a null vector $\zeta$, and a function $\mu$ of one variable as mentioned in Theorem 2 .

Case (II): Suppose that the second fundamental form takes the form (5.3) with respect to a suitable pseudo-orthonormal frame $\left\{E_{1}, \ldots, E_{n}\right\}$ satisfying (5.2).

In this case, from (5.2) and (5.3) and Codazzi's equation, we obtain

$$
\begin{aligned}
& \omega_{2}^{1}\left(E_{3}\right)=\omega_{2}^{4}\left(E_{3}\right)=\cdots=\omega_{2}^{n}\left(E_{3}\right)=0, \\
& \omega_{i}^{1}\left(E_{3}\right)=\omega_{3}^{1}\left(E_{i}\right)=\omega_{2}^{3}\left(E_{i}\right)=\omega_{i}^{1}\left(E_{j}\right)=\omega_{i}^{3}\left(E_{j}\right)=0, \quad i, j \neq 1,3, \\
& \omega_{2}^{k}\left(E_{i}\right)=0, \quad 4 \leq k \leq n .
\end{aligned}
$$

Thus, $\operatorname{Span}\left\{E_{2}, E_{3}\right\}$ and $\operatorname{Span}\left\{E_{2}, E_{4}, \ldots, E_{n}\right\}$ are integrable distributions and their leaves are linear subspaces of $L^{n}$ and thus $\left\{E_{2}, E_{3}, \ldots, E_{n}\right\}$ gives rise to a linear subspace too. This means that we can still parameterize $L^{n}$ such that

$$
x\left(t, v_{2}, \ldots, v_{n}\right)=\gamma(t)+\sum_{i=2}^{n} v_{i} \gamma_{i}(t),
$$

such that $\partial / \partial t=E_{1}$ and $\operatorname{Span}\left\{\partial / \partial v_{2}, \ldots, \partial / \partial v_{n}\right\}=\operatorname{Span}\left\{E_{2}, \ldots, E_{n}\right\}$.

We now consider the following change of basis. We take functions $a_{3}, a_{4}, \ldots, a_{n}$ and define $F_{1}, \ldots, F_{n}$ by (5.5). Unfortunately, the expression for the second fundamental form is not preserved under such a change. However, we still have

$$
\begin{aligned}
& h\left(F_{1}, F_{1}\right) \in \operatorname{Span}\left\{J F_{2}, J F_{3}\right\}, \\
& h\left(F_{1}, F_{3}\right) \in \operatorname{Span}\left\{J F_{2}\right\},
\end{aligned}
$$

and all other components still vanish.

Since $F_{1}$ is a null vector field, there exist suitable functions $b_{3}, \ldots, b_{n}$ such that $F_{1}$ is parallel to some constant null vector, say $\zeta$. In particular, this implies that an integral curve $\gamma=\gamma(t)$ of $F_{1}$ is a null line parallel to $\zeta$ in $L^{n}$. So, by reparameterizing, we may assume $\gamma(t)=t \zeta$ for some null vector $\zeta$.

Taking now $\tilde{F}_{2}$ as the multiple of $F_{2}$ such that $\left\langle\zeta, \tilde{F}_{2}\right\rangle=1$, it follows from (5.14) that

$$
\nabla_{F_{i}} \tilde{F}_{2}=0
$$


for $2 \leq i \leq n$. This means that in the $(n-1)$-dimensional subspace mentioned before, $\tilde{F}_{2}$ is a constant. It only changes along the integral curve of $F_{1}$. It follows that we may write

$$
x\left(t, u_{2}, \ldots, u_{n}\right)=t \zeta+u_{2} \alpha_{2}(t)+\cdots+u_{n} \alpha_{n}(t),
$$

where $\partial / \partial u_{2}=\tilde{F}_{2}$ and $\operatorname{Span}\left\{\partial / \partial u_{2}, \ldots, \partial / \partial u_{n}\right\}=\operatorname{Span}\left\{F_{2}, \ldots, F_{n}\right\}$ and thus $\alpha_{2}(t), \ldots$, $\alpha_{n}(t)$ satisfy

$$
\left\{\begin{array}{l}
\alpha_{2}(t)=b(t) E_{2}, \\
\left\langle\zeta, \alpha_{2}(t)\right\rangle=1, \\
\left\langle\alpha_{2}, \alpha_{2}\right\rangle=0, \\
\left\langle\zeta, \alpha_{i}(t)\right\rangle=\left\langle\alpha_{2}(t), \alpha_{i}(t)\right\rangle=0, \\
\left\langle\alpha_{i}(t), \alpha_{j}(t)\right\rangle=\delta_{i j}, \quad 3 \leq i, j \leq n
\end{array}\right.
$$

Here we also choose again $\left\{\alpha_{3}, \ldots, \alpha_{n}\right\}$ to be the Frenet frame of the null curve $\alpha=\alpha_{2}(t)$ with $\varphi_{3}, \ldots, \varphi_{n}$ to be its corresponding Frenet curvatures as mentioned in Section 4.

From (4.4), (5.3), (5.5), (5.9), (5.16) and the total symmetry of $\langle h(X, Y), J Z\rangle$ we get

$$
\begin{aligned}
& h\left(\frac{\partial}{\partial t}, \frac{\partial}{\partial t}\right)=\beta_{2} J\left(\frac{\partial}{\partial u_{2}}\right)+\sum_{i=3}^{n} g_{12} \beta_{i} J\left(\frac{\partial}{\partial u_{i}}\right), \\
& h\left(\frac{\partial}{\partial t}, \frac{\partial}{\partial u_{k}}\right)=\beta_{k} J\left(\frac{\partial}{\partial u_{2}}\right), \quad 3 \leq k \leq n, \\
& h\left(\frac{\partial}{\partial t}, \frac{\partial}{\partial u_{2}}\right)=h\left(\frac{\partial}{\partial u_{i}}, \frac{\partial}{\partial u_{j}}\right)=0, \quad 2 \leq i, j \leq n,
\end{aligned}
$$

for some functions $\beta_{2}, \ldots, \beta_{n}$.

From (5.17), Codazzi equation (2.6) with $X=\partial / \partial u_{k}, k \geq 3, Y=\partial / \partial u_{2}$ and $Z=\partial / \partial t$, we obtain

$$
\frac{\partial \beta_{k}}{\partial u_{2}}=\beta_{k}\left(\Gamma_{21}^{1}-\Gamma_{22}^{2}\right), \quad 3 \leq k \leq n .
$$

From (5.17), Codazzi equation (2.6) with $X=Z=\partial / \partial t$ and $Y=\partial / \partial u_{2}$, we obtain

$$
\frac{\partial \beta_{2}}{\partial u_{2}}=\beta_{2}\left(\Gamma_{21}^{1}-\Gamma_{22}^{2}\right)-g_{12} \sum_{i=3}^{n} \beta_{i} \Gamma_{2 i}^{2}+\sum_{j=3}^{n} \Gamma_{12}^{j} \beta_{j} .
$$

From (5.17), Codazzi equation (2.6) with $X=Z=\partial / \partial t$ and $Y=\partial / \partial u_{k}, k \geq 3$, we obtain

$$
\begin{aligned}
\frac{\partial \beta_{2}}{\partial u_{k}} & +\beta_{2}\left(\Gamma_{k 2}^{2}-\Gamma_{k 1}^{1}\right)+g_{12} \sum_{i=3}^{m} \beta_{i} \Gamma_{k i}^{2}-\sum_{j=3}^{n} \beta_{j} \Gamma_{k 1}^{j} \\
= & \frac{\partial \beta_{k}}{\partial t}+\beta_{k}\left(\Gamma_{12}^{2}-\Gamma_{11}^{1}\right), \quad 3 \leq k \leq n .
\end{aligned}
$$

From (5.17), Codazzi equation (2.6) with $X=\partial / \partial t, Y=\partial / \partial u_{k}$ and $Z=\partial / \partial u_{j}, j, k \geq 3$, we obtain

$$
\frac{\partial \beta_{j}}{\partial u_{k}}=-\beta_{k} \Gamma_{1 j}^{1}-\beta_{j} \Gamma_{k 2}^{2}-\beta_{2} \Gamma_{k j}^{1}, \quad j, k \geq 3
$$


Using (4.7), equations (5.18) through (5.21) reduce to

$$
\frac{\partial \beta_{k}}{\partial u_{2}}=0, \quad 3 \leq k \leq n
$$

$$
\begin{gathered}
\frac{\partial \beta_{2}}{\partial u_{2}}=\varphi_{3} \beta_{3}, \\
\frac{\partial \beta_{2}}{\partial u_{k}}=\frac{\partial \beta_{k}}{\partial t}-\beta_{k} \Gamma_{11}^{1}+\beta_{2} \Gamma_{k 1}^{1}+\sum_{j=3}^{n} \beta_{j} \Gamma_{k 1}^{j}, \quad 3 \leq k \leq n, \\
\frac{\partial \beta_{3}}{\partial u_{k}}=\frac{\beta_{k} \varphi_{3}}{1-u_{3} \varphi_{3}}, \quad 3 \leq k \leq n, \\
\frac{\partial \beta_{j}}{\partial u_{k}}=0, \quad 4 \leq j \leq n, \quad 3 \leq k \leq n .
\end{gathered}
$$

From (5.22) and (5.26) we obtain

$$
\beta_{4}=\beta_{4}(t), \ldots, \beta_{n}=\beta_{n}(t) .
$$

Also, from (5.22) we obtain

$$
\beta_{3}=\beta_{3}\left(t, u_{3}, \ldots, u_{n}\right) .
$$

Hence, by solving (5.25) for $k \geq 4$, we get

$$
\beta_{3}=b_{3}\left(t, u_{3}\right)+\varphi_{3} \sum_{k=4}^{n} \frac{\beta_{k}}{1-u_{3} \varphi_{3}} u_{k}
$$

for some function $b_{3}\left(t, u_{3}\right)$. Thus, by solving (5.25) with $k=3$, we find

$$
\beta_{3}=\frac{\lambda(t)+\varphi_{3} \sum_{k=4}^{n} \beta_{k} u_{k}}{1-u_{3} \varphi_{3}}
$$

for some function $\lambda=\lambda(t)$.

From (4.7), (5.27) and (5.24) with $k=n$, we get

$$
\frac{\partial \beta_{2}}{\partial u_{n}}=\beta_{n}^{\prime}(t)-\beta_{n-1} \varphi_{n}+\frac{\beta_{n}}{1-u_{3} \varphi_{3}}\left(u_{3} \varphi_{3}^{\prime}+u_{2} \varphi_{3}^{2}-u_{4} \varphi_{3} \varphi_{4}\right) .
$$

Solving (5.31) yields

$$
\begin{aligned}
\beta_{2}= & \left\{\beta_{n}^{\prime}(t)-\beta_{n-1} \varphi_{n}+\frac{\beta_{n}}{1-u_{3} \varphi_{3}}\left(u_{3} \varphi_{3}^{\prime}+u_{2} \varphi_{3}^{2}-u_{4} \varphi_{3} \varphi_{4}\right)\right\} u_{n} \\
& +F_{1}\left(t, u_{2}, \ldots, u_{n-1}\right)
\end{aligned}
$$

for some function $F_{1}=F_{1}\left(t, u_{2}, \ldots, u_{n-1}\right)$. 
Similarly, by solving (5.24) for $k=5, \ldots, n-1$ and applying (4.7), (5.27) and (5.32), we may obtain

$$
\begin{aligned}
\beta_{2}= & \sum_{j=5}^{n}\left\{\beta_{j}^{\prime}(t)-\beta_{j-1} \varphi_{j}+\frac{\beta_{j}}{1-u_{3} \varphi_{3}}\left(u_{3} \varphi_{3}^{\prime}+u_{2} \varphi_{3}^{2}-u_{4} \varphi_{3} \varphi_{4}\right)\right\} u_{j} \\
& +\sum_{j=6}^{n} \beta_{j} \varphi_{j} u_{j-1}+F\left(t, u_{2}, u_{3}, u_{4}\right)
\end{aligned}
$$

for some function $F=F\left(t, u_{2}, u_{3}, u_{4}\right)$. From (5.33) we get

$$
\frac{\partial \beta_{2}}{\partial u_{4}}=\frac{\partial F}{\partial u_{4}}-\frac{\varphi_{3} \varphi_{4}}{1-u_{3} \varphi_{3}} \sum_{j=5}^{n} \beta_{j} u_{j} .
$$

On the other hand, from (5.24) with $k=4$ together with (4.7) and (5.27), we find

$$
\begin{aligned}
\frac{\partial \beta_{2}}{\partial u_{4}}= & \beta_{4}^{\prime}(t)-\frac{\lambda \varphi_{4}+\varphi_{3} \varphi_{4} \sum_{k=4}^{n} \beta_{k} u_{k}}{1-u_{3} \varphi_{3}} \\
& +\frac{\beta_{4}}{1-u_{3} \varphi_{3}}\left(u_{3} \varphi_{3}^{\prime}+u_{2} \varphi_{3}^{2}-u_{4} \varphi_{3} \varphi_{4}\right)+\beta_{5} \varphi_{5} .
\end{aligned}
$$

Combining (5.34) and (5.35), we obtain

$$
\frac{\partial F}{\partial u_{4}}=\beta_{4}^{\prime}(t)-\frac{\lambda \varphi_{4}}{1-u_{3} \varphi_{3}}+\frac{\beta_{4}}{1-u_{3} \varphi_{3}}\left(u_{3} \varphi_{3}^{\prime}+u_{2} \varphi_{3}^{2}-u_{4} \varphi_{3} \varphi_{4}\right)+\beta_{5} \varphi_{5} .
$$

Thus, by solving (5.36) and by using (5.33), we obtain

$$
\begin{aligned}
\beta_{2}= & \sum_{j=4}^{n}\left\{\beta_{j}^{\prime}(t)-\beta_{j-1} \varphi_{j}+\frac{\beta_{j}}{1-u_{3} \varphi_{3}}\left(u_{3} \varphi_{3}^{\prime}+u_{2} \varphi_{3}^{2}-u_{4} \varphi_{3} \varphi_{4}\right)\right\} u_{j} \\
& +\beta_{3} \varphi_{4} u_{4}+\sum_{j=5}^{n} \beta_{j} \varphi_{j} u_{j-1}-\frac{\lambda \varphi_{4}}{1-u_{3} \varphi_{3}} u_{4}+L\left(t, u_{2}, u_{3}\right)
\end{aligned}
$$

for some function $L=L\left(t, u_{2}, u_{3}\right)$.

From (5.24) with $k=3$ we have

$$
\frac{\partial \beta_{2}}{\partial u_{3}}=\beta_{3}^{\prime}(t)+\frac{\beta_{3}}{g_{12}}\left(u_{3} \varphi_{3}^{\prime}+2 \varphi_{3} g_{13}\right)-\frac{\beta_{2} \varphi_{3}}{g_{12}}+\beta_{4} \varphi_{4}+\frac{\varphi_{3}}{g_{12}} \sum_{k=4}^{n} \beta_{k} g_{1 k},
$$

where $g_{12}, \ldots, g_{1 n}$ are given by (4.5). Substituting (5.30) into (5.38) yields

$$
\begin{aligned}
\frac{\partial \beta_{2}}{\partial u_{3}}= & \beta_{4} \varphi_{4}+\frac{1}{g_{12}}\left\{\lambda^{\prime}+\varphi_{3}^{\prime} \sum_{k=4}^{n} \beta_{k} u_{k}+\varphi_{3} \sum_{k=4}^{n} \beta_{k}^{\prime} u_{k}+2 \beta_{3} \varphi_{3}^{\prime} u_{3}\right. \\
& \left.+2 \beta_{3} \varphi_{3} g_{13}+\varphi_{3} \sum_{k=4}^{n} \beta_{k} g_{1 k}-\beta_{2} \varphi_{3}\right\} .
\end{aligned}
$$


Substituting (5.37) into (5.39) yields

$$
\frac{\partial L}{\partial u_{3}}=\beta_{4} \varphi_{4}+\frac{1}{g_{12}}\left\{\lambda^{\prime}+\varphi_{3} \varphi_{4} \beta_{4} u_{3}-\varphi_{3} L\right\}+\frac{2 \lambda}{g_{12}^{2}}\left\{\varphi_{3}^{\prime} u_{3}+\varphi_{3}^{2} u_{2}\right\} .
$$

Since $g_{12}=1-\varphi_{3} u_{3},(5.40)$ is a first order linear differential equation. By solving (5.40), we obtain

$$
L=\mu\left(t, u_{2}\right) g_{12}+\frac{\lambda^{\prime}}{\varphi_{3}}+\frac{\lambda \varphi_{3}^{\prime}}{\varphi_{3}^{2} g_{12}}\left(2 \varphi_{3} u_{3}-1\right)+\frac{\varphi_{4} \beta_{4}}{\varphi_{3}}+\frac{\lambda \varphi_{3} u_{2}}{g_{12}}
$$

for some function $\mu=\mu\left(t, u_{2}\right)$. Combining (5.41) and (5.37) yields

$$
\begin{aligned}
\beta_{2}= & \sum_{j=4}^{n}\left\{\beta_{j}^{\prime}(t)-\beta_{j-1} \varphi_{j}+\frac{\beta_{j}}{1-u_{3} \varphi_{3}}\left(u_{3} \varphi_{3}^{\prime}+u_{2} \varphi_{3}^{2}-u_{4} \varphi_{3} \varphi_{4}\right)\right\} u_{j} \\
& +\beta_{3} \varphi_{4} u_{4}+\sum_{j=5}^{n} \beta_{j} \varphi_{j} u_{j-1}-\frac{\lambda \varphi_{4}}{1-u_{3} \varphi_{3}} u_{4} \\
& +\mu g_{12}+\frac{\lambda^{\prime}}{\varphi_{3}}+\frac{\lambda \varphi_{3}^{\prime}}{\varphi_{3}^{2} g_{12}}\left(2 \varphi_{3} u_{3}-1\right)+\frac{\varphi_{4} \beta_{4}}{\varphi_{3}}+\frac{\lambda \varphi_{3} u_{2}}{g_{12}} .
\end{aligned}
$$

From (5.23), (5.30) and (5.42) we get $\partial \mu / \partial u_{2}=0$. Thus, $\mu=\mu(t)$. Equation (5.42) is nothing but (4.12). Hence, the second fundamental form of the immersion $\Psi$ of $L^{n}$ into $\boldsymbol{C}_{1}^{n}$ satisfies (4.11), (4.12) and (4.13), which coincides with the second fundamental form of $F_{\alpha \zeta \mu \lambda \beta_{4} \cdots \beta_{n}}$. Therefore, by applying Theorem B of uniqueness, we conclude that, up to rigid motions of $\boldsymbol{C}_{1}^{n}$, the immersion $\Psi$ is given by a $F_{\alpha \zeta \mu \lambda \beta_{4} \cdots \beta_{n}}$.

Finally, if $\Psi: L^{n} \rightarrow \boldsymbol{C}_{1}^{n}$ is a totally geodesic Lagrangian submanifold, then $\Psi$ is nothing but a $F_{\alpha \zeta \mu}: L^{n} \rightarrow C_{1}^{n}$ with $\mu=0$ according to Theorem 2.

6. Explicit examples. In this section we provide some explicit expression of flat Lagrangian minimal submanifolds in $\boldsymbol{C}_{1}^{n}$.

EXAMPLE 6.1. Let $\mu=\mu(t)$ be a function, $\gamma(t)=t \zeta$ a null line in $L^{n}$, and $\alpha=\alpha(t)$ a null line lying in the hyperplane $\Lambda^{n-1}=\left\{x \in L^{n}:\langle x, \zeta\rangle=1\right\}$. Choose $\alpha_{3}, \ldots, \alpha_{n}$ to be an orthonormal basis of $\{\zeta, \alpha\}^{\perp}$. Then all of Christoffel's symbols of $L^{n}$ with respect to the parameterization (4.2) are equal to zero. Hence, from (4.3) and Gauss' formula, we know that the position function $x$ of the immersion $F_{\alpha \zeta \mu}: L^{n} \rightarrow \boldsymbol{C}_{1}^{n}$ satisfies

$$
\begin{aligned}
& \frac{\partial^{2} x}{\partial t^{2}}=-\mu(t) i \frac{\partial x}{\partial u_{2}}, \\
& \frac{\partial^{2} x}{\partial t \partial u_{j}}=\frac{\partial^{2} x}{\partial u_{j} \partial u_{k}}=0, \quad 2 \leq j, k \leq n .
\end{aligned}
$$


By solving the partial differential system (6.1), we conclude that the corresponding minimal Lagrangian isometric immersion $F_{\alpha \zeta \mu}: L^{n} \rightarrow C_{1}^{n}$ is given by

$$
\begin{aligned}
x\left(t, u_{2}, \ldots, u_{n}\right)=\frac{1}{2}\left(t+u_{2}-i \int^{t} \int^{s} \mu(s) d s d t,\right. & \left.t-u_{2}+i \int^{t} \int^{s} \mu(s) d s d t, 2 u_{3}, \ldots, 2 u_{n}\right),
\end{aligned}
$$

where we choose the initial conditions as

$$
\begin{gathered}
x(0)=(0, \ldots, 0), \frac{\partial x}{\partial t}(0)=\left(\frac{1}{2}, \frac{1}{2}, 0, \ldots, 0\right), \frac{\partial x}{\partial u_{2}}(0)=\left(\frac{1}{2},-\frac{1}{2}, 0, \ldots, 0\right), \\
\frac{\partial x}{\partial u_{3}}(0)=(0,0,1,0, \ldots, 0), \ldots, \frac{\partial x}{\partial u_{n}}(0)=(0, \ldots, 0,1) .
\end{gathered}
$$

EXAMPLE 6.2. Let $\gamma(t)=t \zeta$ be a null line in $L^{n}$, and $\alpha=\alpha(t)$ a null curve lying in the hyperplane $\Lambda^{n-1}=\left\{x \in L^{n} ;\langle x, \zeta\rangle=1\right\}$ with Frenet curvatures $\varphi_{3}=1, \varphi_{4}=$ $\cdots=\varphi_{n}=0$. Choose $\mu=1$. Then the Christoffel symbols of $L^{n}$ with respect to the parameterization (4.2) are given by

$$
\begin{aligned}
& \Gamma_{11}^{1}=\Gamma_{12}^{2}=-\Gamma_{13}^{3}=\frac{u_{2}}{u_{3}-1}, \quad \Gamma_{11}^{3}=\frac{u_{2}^{2}}{1-u_{3}}, \quad \Gamma_{13}^{1}=\frac{1}{u_{3}-1}, \\
& \Gamma_{12}^{3}=1, \quad \Gamma_{j k}^{i}=0, \quad \text { otherwise. }
\end{aligned}
$$

From (4.3), (6.4) and Gauss' formula, we know that the position function $x$ of the immersion $F_{\alpha \zeta \mu}: L^{n} \rightarrow \boldsymbol{C}_{1}^{n}$ satisfies

$$
\begin{aligned}
& \frac{\partial^{2} x}{\partial t^{2}}=\frac{u_{2}}{u_{3}-1} \frac{\partial x}{\partial t}+\frac{u_{2}^{2}}{1-u_{3}} \frac{\partial x}{\partial u_{3}}+\left(u_{3}-1\right) i \frac{\partial x}{\partial u_{2}}, \\
& \frac{\partial^{2} x}{\partial t \partial u_{2}}=\frac{\partial x}{\partial u_{3}}, \quad \frac{\partial^{2} x}{\partial t \partial u_{3}}=\frac{1}{u_{3}-1} \frac{\partial x}{\partial t}+\frac{u_{2}}{1-u_{3}} \frac{\partial x}{\partial u_{3}}, \\
& \frac{\partial^{2} x}{\partial t \partial u_{r}}=\frac{\partial^{2} x}{\partial u_{j} \partial u_{k}}=0, \quad 4 \leq r \leq n, \quad 2 \leq j, k \leq n .
\end{aligned}
$$

By solving the partial differential system (6.5), we conclude that the corresponding minimal Lagrangian isometric immersion $F_{\alpha \zeta \mu}: L^{n} \rightarrow \boldsymbol{C}_{1}^{n}$ is given by

$$
\begin{gathered}
x\left(t, u_{2}, \ldots, u_{n}\right)=\frac{1}{6}\left(2\left(u_{2}+i-u_{3} i\right) e^{-i t}+\left(u_{3} i-i+\frac{u_{2}}{2}(1+\sqrt{3} i)\right) e^{(\sqrt{3}+i) t / 2}\right. \\
+\left(u_{3} i-i+\frac{1}{2}(1-\sqrt{3} i) u_{2}\right) e^{(-\sqrt{3}+i) t / 2},
\end{gathered}
$$

$$
\begin{aligned}
& \sqrt{3}\left(1-u_{3}-\frac{u_{2}}{2}(\sqrt{3}-i)\right) e^{(\sqrt{3}+i) t / 2}+\sqrt{3}\left(u_{3}-1-\frac{u_{2}}{2}(\sqrt{3}+i)\right) e^{(-\sqrt{3}+i) t / 2} \\
& 2\left(u_{3}-1+i u_{2}\right) e^{-i t}+\left(2 u_{3}-2+(\sqrt{3}-i) u_{2}\right) e^{(\sqrt{3}+i) t / 2} \\
& \left.\quad+\left(2 u_{3}-2-(\sqrt{3}+i) u_{2}\right) e^{(-\sqrt{3}+i) t / 2}, 6 u_{4}, \ldots, 6 u_{n}\right)
\end{aligned}
$$


where we choose the initial conditions given by (6.3)

EXAMPLE 6.3. Let $\gamma(t)=t \zeta$ be a null line in $L^{n}$, and $\alpha=\alpha(t)$ a null curve lying in the hyperplane $\Lambda^{n-1}=\left\{x \in L^{n} ;\langle x, \zeta\rangle=1\right\}$ with Frenet curvatures $\varphi_{3}=1, \varphi_{4}=\cdots=$ $\varphi_{n}=0$. Choose $\lambda=1$ and $\beta_{4}=\cdots=\beta_{n}=0$. Then the Christoffel symbols of $L^{n}$ with respect to the parameterization (4.2) are given by (6.4). Hence, from (4.11) and Gauss' formula, we know that the position function $x$ of the immersion $I_{\alpha \zeta \mu \lambda \beta_{4} \cdots \beta_{n}}: L^{n} \rightarrow \boldsymbol{C}_{1}^{n}$ satisfies

$$
\begin{aligned}
& \frac{\partial^{2} x}{\partial t^{2}}=\frac{u_{2}}{u_{3}-1} \frac{\partial x}{\partial t}+\frac{u_{2}^{2}}{1-u_{3}} \frac{\partial x}{\partial u_{3}}+\frac{u_{2} i}{1-u_{3}} \frac{\partial x}{\partial u_{2}}+i \frac{\partial x}{\partial u_{3}}, \\
& \frac{\partial^{2} x}{\partial t \partial u_{2}}=\frac{\partial x}{\partial u_{3}}, \quad \frac{\partial^{2} x}{\partial t \partial u_{3}}=\frac{1}{u_{3}-1} \frac{\partial x}{\partial t}+\frac{u_{2}}{1-u_{3}} \frac{\partial x}{\partial u_{3}}+\frac{i}{1-u_{3}} \frac{\partial x}{\partial u_{2}}, \\
& \frac{\partial^{2} x}{\partial t \partial u_{r}}=\frac{\partial^{2} x}{\partial u_{j} \partial u_{k}}=0, \quad 4 \leq r \leq n, \quad 2 \leq j, k \leq n .
\end{aligned}
$$

By solving the partial differential system (6.7), we conclude that the corresponding minimal Lagrangian isometric immersion $I_{\alpha \zeta \mu \lambda \beta_{4} \cdots \beta_{n}}: L^{n} \rightarrow C_{1}^{n}$ is given by

$$
\begin{aligned}
x\left(t, u_{2}, \ldots, u_{n}\right)= & \frac{1}{2}\left(i t+u_{2}+(i-1)\left(u_{3} t-t+\frac{1}{2} t^{2} u_{2}+\frac{1}{6} i t^{3}\right),\right. \\
& -\left(i t+u_{2}+(i+1)\left(u_{3} t-t+\frac{1}{2} t^{2} u_{2}+\frac{1}{6} i t^{3}\right)\right), \\
& \left.2 t u_{2}+i t^{2}+2 u_{3}, 2 u_{4}, \ldots, 2 u_{n}\right),
\end{aligned}
$$

where we choose the initial conditions given by (6.3).

REMARK 6.1. When $n=2$, Theorem 4 implies that locally, up to rigid motions of $\boldsymbol{C}_{1}^{2}$, every minimal Lagrangian isometric immersion of $L^{2}$ into $\boldsymbol{C}_{1}^{2}$ must be given by a $F_{\alpha \zeta \mu}$, where $\zeta$ is a null vector, $\alpha$ a null line in $\Lambda^{1}$ and $\mu$ a function of $t$, respectively. Hence, from Example 6.1, we obtain the following.

COROLLARY 5. Locally, up to rigid motions of $\boldsymbol{C}_{1}^{2}$, a minimal Lagrangian isometric immersion $\Phi: L^{2} \rightarrow C_{1}^{2}$ of $L^{2}$ into $C_{1}^{2}$ is given by

$$
x\left(t, u_{2}\right)=\frac{1}{2}\left(t+u_{2}-i \int^{t} \int^{s} \mu(s) d s d t, t-u_{2}+i \int^{t} \int^{s} \mu(s) d s d t\right) .
$$

\section{REFERENCES}

[BR] M. BARros And A. Romero, Indefinite Kähler manifolds, Math. Ann. 261 (1982), 55-62.

[C1] B. Y. CHEN, Interaction of Legendre curves and Lagrangian submanifolds, Israel J. Math. 99 (1977), 69-108.

[C2] B. Y. CHEN, Complex extensors and Lagrangian submanifolds in complex Euclidean spaces, Tôhoku Math. J. 49 (1997), 277-297.

[C3] B. Y. CHEN, Representation of flat Lagrangian $H$-umbilical submanifolds in complex Euclidean spaces, Tôhoku Math. J. 51 (1999), 13-20. 
[CDVV1] B. Y. Chen, F. Dillen, L. Verstraelen and L. VRancken, An exotic totally real minimal immersions of $S^{3}$ into $C P^{3}$ and its characterization, Proc. Roy. Soc. Edinburgh Sec. A 126 (1996), $153-165$.

[CDVV2] B. Y. Chen, F. Dillen, L. Verstraelen and L. VRancken, Lagrangian isometric immersions of a real-space-form $M^{n}(c)$ into a complex-space-form $\tilde{M}^{n}(4 c)$, Math. Proc. Cambridge Philos. Soc. 124 (1998), 107-125.

[CO] B. Y. CHEn And K. OGIUE, On totally real submanifolds, Trans. Amer. Math. Soc. 193 (1974), 257266.

[E] N. EJIRI, Totally real minimal immersions of $n$-dimensional real space forms into $n$-dimensional complex space forms, Proc. Amer. Math. Soc. 84 (1982), 243-246.

[KV1] M. KRIELE AND L. VRANCKEN, Lorentzian affine hyperspheres with constant sectional curvature, Trans. Amer. Math. Soc. 352 (2000), 1581-1599.

[KV2] M. KRIELE AND L. VRANCKEn, Minimal Lagrangian submanifolds of Lorentzian complex space forms with constant sectional curvature, Arch. Math. 72 (1999), 223-232.

[O] K. OGiUe, Some recent topics in the theory of submanifolds, Sugaku Expositions 4 (1991), 21-41.

[On] B. O'NEILL, Semi-Riemannian geometry with applications to relativity, Academic Press, New York (1983).

DEPARTMENT OF MATHEMATICS Michigan STATE UNIVERSITY

EAST LANSING

MICHIGAN 48824-1027

USA
MATHEMATISCH INSITUTUUT

UNIVERSITEIT UTRECHT

P.O. BoX 80.010

3508 TA UTRECHT

THE NETHERLANDS 